Rev. salud pública. 6 (2): 199-211, 2004

\title{
Plaguicidas usados en la Fumigación de Cultivos Ilícitos y Salud Humana: ¿una Cuestión de Ciencia o Política?
}

\author{
Alvaro Javier Idrovo \\ Médico, Especialista en Higiene y Salud Ocupacional, M. Sc. Salud Pública, M. Sc. \\ Salud Ambiental. Ph D (candidato) en Epidemiología. Instituto Nacional de Salud \\ Pública. Cuernavaca, Morelos, México. \\ E-mail: ajidrovo@espm.insp.mx; idrovoaj@hotmail.com
}

Recibido 5 Marzo 2004/Enviado para Modificación 4 Mayo 2004/Aprobado 29 Mayo 2004

\section{RESUMEN}

Objetivo Los potenciales efectos adversos sobre la salud humana por el uso de plaguicidas en el programa de erradicación de cultivos ilícitos en Colombia es un tema controversial. Desde comienzos de la década de los 80 se han usado, en programas de erradicación o experimentalmente, los plaguicidas paraquat, triclopir, imazapir, glifosato y tebutiuron. El objetivo del estudio es identificar que aproximación razonable podría adoptar la salud pública acerca de esta problemática.

Métodos Se realizó un análisis cualitativo del contenido de los discursos de los actores sociales involucrados en este debate, con el fin de clasificarlos de acuerdo con las expectativas, o aproximación lega al riesgo, de Suppes. Los actores sociales fueron identificados mediante los documentos disponibles en medio físico o Internet; posteriormente, de éstos se seleccionaron y analizaron los textos ideales-típicos.

Resultados Las lesiones dérmicas, conjuntivitis e infecciones gastrointestinales y respiratorias fueron identificadas como las dolencias más frecuentes. El análisis de los discursos identifica a los grupos afectados como poblaciones vulnerables; los gobiernos colombiano y estadounidense presentan informes, basados en el "modelo médico", que señalan no existir asociación entre la exposición a glifosato y salud humana; la guerrilla no señala una clara relación entre plaguicidas y efectos sobre la salud; la comunidad científica, nacional e internacional, critica el programa de erradicación con base en conceptos de la ecología y la Medicina Social Latinoamericana; los grupos ambientalistas critican cualquier uso de plaguicidas, y los defensores de derechos humanos señalan que las fumigaciones violan las leyes colombianas y estadounidenses. 
Discusión Se concluye que las diferentes posturas son irreconciliables y que ninguna puede considerarse irrefutable. Una postura razonable para la salud pública es apoyar la adopción del principio de precaución.

Palabras Claves: Plaguicidas, intoxicación, ciencia, política de salud, Colombia (fuente:DECS, BIREME).

\section{ABSTRACT \\ Pesticides used in the spraying of illicit crops and human health: An issue of science or politics?}

Objective The potential adverse effects on human health associated with pesticides used in the program of eradication of illicit crops in Colombia are controversial. From the beginning of the 80's the pesticides paraquat, triclopyr, imazapyr, glyphosate and tebuthiuron have been used in eradication programs or experimentally. The objective of this study was to identify a reasonable approach which could be adopted by public health on this problem.

Methods A qualitative content analysis was carried out of the speeches of social actors involved in this debate, to classify them according to Suppes' expectations, or lay approach to risk. Social actors were identified by means of available documents in hard copies or on the internet; afterwards, ideally typical texts were selected and analysed.

Results Skin lesions, conjunctivitis, and gastrointestinal and respiratory infections were identified as the most frequent illnesses. The analysis of speeches identified the affected groups as vulnerable populations. The Colombian and American governments present reports, based on the "medical model", which indicate no association between glyphosate exposure and human health. Guerrilla groups do not show a clear relationship between pesticides and effects on health; the national and international scientific communities criticize the eradication program based on concepts from ecology and the Latin American Social Medicine; environmentalist groups criticize any use of pesticides, and human right defenders point out that sprayings violate Colombian and American laws.

Discussion In conclusion the different positions are opposed and none can be considered irrefutable. A rational position from the public health standpoint is to support the adoption of the precaution principle.

Key Words: Pesticides, poisoning, science, health policy, Colombia (source: $\mathrm{MeSH}, \mathrm{NLM})$.

$\mathrm{E}$ n Colombia se cultiva marihuana desde la década de los 60. Durante esta época no se usaron plaguicidas para su erradicación, debido a la polémica en Estados Unidos de América por el potencial efecto nocivo que traería a los consumidores de marihuana la exposición a residuos de 
paraquat, tal como había sucedido en México (1). Hacia 1980 ya se habían hecho ensayos experimentales con paraquat, pero sólo en 1984 se aprobó el uso de plaguicidas para la erradicación de cultivos ilícitos. Sin embargo, para evitar la controversia que ocasionaría el uso del paraquat se eligió al glifosato, por su menor toxicidad (2). El uso de glifosato en la costa atlántica colombiana suscitó protestas por parte de grupos indígenas, campesinos y asociaciones científicas; pese a estas críticas iniciales se siguió fumigando, logrando reducir las áreas cultivadas. Ese aparente éxito no fue duradero, y en 1986 Colombia volvió a ser el primer país exportador de marihuana hacia EUA, al trasladar las zonas de cultivo al suroriente del país (2).

A finales de 1985 se decidió usar el plaguicida Garlon $4{ }^{\circledR}$ (ingrediente activo: triclopir) para erradicar los cultivos de coca, pero fue retirado debido a que se consideró peligroso y la compañía productora rehusó proveerlo al gobierno colombiano (1). Para aquella época el organismo gubernamental ambiental, Inderena, informaba sobre efectos adversos en el Parque Nacional Tairona, debidos al uso de glifosato (2). Pese a estas denuncias, desde comienzos de 1992 las estrategias de fumigación con glifosato se expandieron a los cultivos de amapola y, básicamente, son las mismas que actualmente se siguen. La mezcla química usada en el presente tiene el nombre comercial Roundup-Ultra ${ }^{\circledR}$, y está compuesta por glifosato, Cosmoflux $411 \mathrm{~F}^{\circledR}$ (aceite mineral y surfactantes no ionizados con agentes de acoplamiento) y el surfactante POEA (3). Hubo intentos de modificar la estrategia con la inclusión del imazapir, y el hongo Fusarium oxysporum; sin embargo, ninguna de estas modificaciones fue incluida de manera permanente $(1,2)$. Múltiples críticas a este programa de cultivos ilícitos han surgido debido a las características sin precedentes de la fumigación, tales como: i) aplicación de grandes cantidades de plaguicidas, ii) uso de mayores concentraciones que las de las presentaciones comerciales para la agricultura y iii) ausencia de evaluación científica completa de la mezcla usada; las críticas son variadas, e incluyen desde la nocividad para el ambiente, hasta la puesta en duda de la legitimidad del Estado colombiano.

De otro lado, los desarrollos en sociología de la ciencia, análisis de políticas y entendimiento lego de la ciencia y el riesgo, han permitido empezar a entender las diferentes formas de racionalidad de científicos, tomadores de decisiones y el público (4). Cuando estas racionalidades entran en conflicto, como en el caso de los potenciales efectos por la fumigación de los cultivos ilícitos en Colombia, su identificación en el discurso de los actores sociales involucrados puede ser un paso importante para construir acuerdos políticos. Ante esta situación, en este artículo se hace un análisis preliminar de los discursos de los actores sociales involucrados en esta controversia. Este análisis 
pretende ser un aporte, desde las ciencias sociales, para que la salud pública pueda adoptar una postura ante este complejo problema de salud ambiental.

\section{MÉTODOS}

Existen evidencias de que los métodos cualitativos, pese a no ser muy usados en temas ambientales, pueden brindar grandes aportes para comprender este tipo de problemas (5). En este estudio, para cumplir con el objetivo planteado, se realizó una búsqueda de textos disponibles, en medio físico o en la internet, que tuviesen relación con la controversia de potenciales efectos asociados con el programa de erradicación de cultivos ilícitos en Colombia. El análisis del contenido es una forma utilizada por las ciencias sociales para estudiar la naturaleza, los significados fundamentales, los procesos dinámicos y los actores sociales involucrados en un proceso de comunicación (6)

En una primera aproximación se identificaron los actores sociales y, luego, se seleccionaron los documentos que podrían ser los textos ideales-típicos (7) de cada uno de éstos grupos. Finalmente se realizó un análisis crítico de los textos seleccionados, buscando su clasificación dentro de las expectativas de Suppes (8). Esta clasificación identifica cinco tipos de expectativas: i) basadas en un buen conocimiento inductivo de frecuencias aceptadas; ii) basadas en un limitado conocimiento inductivo de frecuencias aceptadas; iii) cuando las frecuencias son cuestionadas; iv) derivadas de otros modelos teóricos, y v) basadas en una mezcla de las anteriores. Según este autor, mediante las expectativas se hace una aproximación al riesgo, desde la forma subjetiva, o lega, de entender el mundo de los diversos actores sociales (8).

\section{RESULTADOS}

En la Tabla 1 se resumen las características de los discursos de los actores sociales estudiados, y a continuación se presentan las características más importantes de cada uno de estos.

Los supuestos efectos adversos: el discurso de los afectados

De acuerdo con numerosas fuentes periodísticas, los directamente afectados señalan múltiples daños asociados con los plaguicidas usados en los programas de erradicación. Los más descritos son dermatosis, conjuntivitis e 
infecciones gastrointestinales y respiratorias $(2,9,10)$. La característica más sobresaliente del discurso de los afectados es la vulnerabilidad de esta población (11); las tres coordenadas de vulnerabilidad de Chambers (12) son evidentes: i) la exposición o el riesgo de ser expuesto a situaciones de crisis: las fumigaciones aéreas; ii) la incapacidad o el riesgo de no tener los recursos necesarios para afrontar estas situaciones: la pobreza y la marginación, y iii) la potencialidad o el riesgo de ser sujeto a consecuencias serias como resultado de las crisis: los efectos adversos sobre la salud.

Tabla 1. Aproximaciones al riesgo de los actores sociales involucrados en la fumigación de cultivos ilícitos en Colombia, 2004

\begin{tabular}{|c|c|c|c|c|c|c|}
\hline \multirow{2}{*}{$\begin{array}{c}\text { Tipo de expectativas } \\
\text { (clasificación de Suppes) }\end{array}$} & \multicolumn{6}{|c|}{ Actores sociales } \\
\hline & 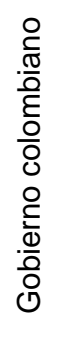 & 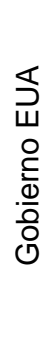 & 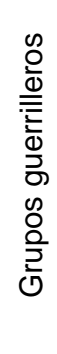 & 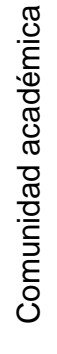 & 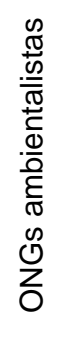 & 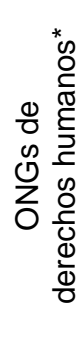 \\
\hline Buen conocimiento inductivo & $\mathrm{X}$ & $x$ & & $\mathrm{X}$ & & \\
\hline $\begin{array}{l}\text { Limitado conocimiento } \\
\text { inductivo }\end{array}$ & $x$ & $\mathrm{X}$ & & $X$ & $X$ & $\mathrm{X}$ \\
\hline Frecuencias cuestionadas & & & & $X$ & $\mathrm{X}$ & $x$ \\
\hline Otros modelos teóricos & & & $x$ & $x$ & $x$ & $x$ \\
\hline
\end{tabular}

Un estudio exploratorio realizado en la frontera colombo-ecuatoriana en 2001, indica que toda la población, en los $5 \mathrm{Km}$ alrededor de las zonas fumigadas, "se han intoxicado con las fumigaciones...", manteniéndose síntomas crónicos como "afecciones neurológicas, problemas de piel y conjuntivas" hasta después de tres meses de haber ocurrido la fumigación (13). Posteriormente, otro estudio similar incluyó a cuatro localidades ecuatorianas y dos colombianas; los hallazgos fueron parecidos, aunque también se reportó una alta ocurrencia de prurito, dermatitis y estrés. Además, en cuatro individuos de la zona cercana a los lugares de fumigación se observó un elevado número de linfocitos alterados en análisis citogenéticos (14). 
$\mathrm{Al}$ analizar estos estudios se observa que las dolencias y hallazgos de laboratorio allí descritos son inespecíficos, y podrían no indicar efectos asociados a los plaguicidas del programa de erradicación. Las definiciones laxas adoptadas pudieron ocasionar un registro exagerado de casos, al aumentar el numerador manteniendo el denominador constante, de acuerdo con la conveniencia de los resultados que se querían hallar (15). Además, otros dos problemas metodológicos son evidentes en estos estudios. El primero se relaciona con la comparación de poblaciones para establecer causalidad $(16,17)$. Para ello los investigadores decidieron escoger como grupo de referencia a los ecuatorianos que viven más lejos de la frontera con Colombia, sin mostrar evidencias que señalen que las poblaciones son comparables. Para definir causalidad es necesario que las poblaciones sean realmente comparables, de manera que las diferencias observadas puedan ser atribuidas a la exposición en estudio (16). El segundo problema es lo no acertado de establecer una relación causal con un estudio de carácter descriptivo (18); el encontrar diferencias estadísticamente significativas no indica una relación causal, especialmente cuando no existe comparabilidad de las poblaciones, lo que es habitual en los estudios observacionales, en los que la asociación por definición se encuentra confundida (16).

La postura oficial

Tanto el gobierno colombiano como el estadounidense han tenido una postura similar. Debido al apoyo estadounidense para combatir el narcotráfico gran parte de los informes del gobierno colombiano se basan en los de funcionarios o agencias norteamericanas, o en estudios realizados por colombianos con financiamiento norteamericano. El "Estudio de las denuncias de daños a la salud relacionadas con la erradicación aérea en Colombia” (19) realizado en un municipio del departamento de Nariño, tuvo por objetivo examinar las denuncias sobre efectos en la salud humana, para lo que se estudiaron los registros de casos clínicos, y se revisaron los datos de vigilancia epidemiológica. Los hallazgos indicaron que la mayoría de los afectados eran menores de 14 años de edad, de sexo femenino, en mal estado nutricional, y cuya principal queja eran las lesiones dérmicas. Como conclusión se señala que "es improbable cualquier nexo de causalidad entre la erradicación aérea con el herbicida glifosato y las afecciones dérmicas...”, ya que estas lesiones eran de origen infeccioso y no pueden estar asociadas con agentes químicos (19). Además, los registros epidemiológicos indicaron que estas afecciones dérmicas se encontraban, desde bastante tiempo atrás, entre las primeras causas de morbilidad en la región. 
Sin embargo, el informe que realmente sirve de sustento a las acciones del programa de erradicación de cultivos ilícitos es un estudio con la metodología de evaluación de riesgos, realizado por la Agencia de Protección Ambiental (EPA) de los EUA. Este trabajo es un extenso informe que incluye la descripción del uso de glifosato en Colombia y los EUA, un resumen de las propiedades del glifosato y sus diferentes formulaciones, la evaluación del riesgo para la salud humana, una revisión de los informes de incidentes con glifosato, y la evaluación ambiental de este herbicida (20). Los resultados más relevantes son que, aunque la formulación de glifosato usada en Colombia no es utilizada en EUA, no debe haber inquietud por los efectos tóxicos del glifosato sobre la salud humana, y que la información disponible es insuficiente para definir con exactitud los efectos sobre el ecosistema.

Los informes anteriores se caracterizan por basarse en el "modelo médico", caracterizado por el método científico positivista, que minimiza las “condiciones basadas en síntomas” (21). El estudio y los registros epidemiológicos son descriptivos, por lo que no pueden definir o descartar relaciones de causalidad; su utilidad para intentar solucionar la problemática es limitada. El informe de la EPA es un estudio técnico con una metodología que frecuentemente se usa cuando se requieren respuestas rápidas ante situaciones en las que no hay estudios con rigor científico, y debe acompañarse de una fase posterior de negociación política entre los diversos actores sociales involucrados. Este último proceso aún no se ha realizado, y el debate aquí descrito podría ser parte de esta negociación. Sin embargo, lo más importante de señalar es que el uso de evidencia científica para justificar el uso de plaguicidas no implica necesariamente que sus conclusiones correspondan a la realidad. Infortunadamente existen muchos ejemplos en los cuales por intereses económicos, ideológicos, emocionales y/o políticos los aportes científicos son usados para justificar algunas políticas cuando los datos son una evidencia inadecuada. Un análisis sobre este tema puede encontrarse en el artículo de Rosenstock \& Lee (22). ¿Esto está ocurriendo en el caso de los plaguicidas usados en la erradicación de cultivos ilícitos en Colombia? Posteriores estudios políticos y sociológicos podrán ayudar a dar una respuesta al respecto.

La aproximación de la guerrilla colombiana

En un comunicado a la opinión pública del 8 de marzo de 2001, de las Fuerzas Armadas Revolucionarias de Colombia (FARC), se puede entender parcialmente la forma en la que este grupo guerrillero entiende la problemática. “Colombia,... necesita resolver su tarea de erradicación de cultivos ilegales 
por la vía de la persuasión y los acuerdos con las comunidades. Los planes unilaterales de fumigación y de violencia tanto química como biológica, lesionan el suelo patrio, nuestra dignidad nacional y solo logran desplazar el problema. Son una agresión... contra el equilibrio ecológico en tanto afectan irreversiblemente la vida humana, animal y vegetal vale decir la biodiversidad, pilar de nuestro futuro...” (23). Al analizar el discurso de la guerrilla se puede observar que no es explícita en indicar cómo los plaguicidas usados en la fumigación de cultivos ilícitos se relacionan con los efectos sobre la salud. Más bien parece ser un discurso con consignas que siguen los objetivos sociales y políticos de una organización guerrillera inconforme con la organización política del país, y con el tipo de relación que tiene Colombia frente a los EUA.

\section{Los discursos de la comunidad científica}

El sector académico también se ha manifestado en contra del uso de plaguicidas en la erradicación de cultivos ilícitos, aunque las posturas han sido muy divergentes. Entre estos sobresalen dos grupos más o menos bien diferenciables; por un lado se encuentran grupos con tendencia biologicista, y por el otro, grupos que pretenden aproximaciones contextuales, con fuerte arraigo con el movimiento de la Medicina Social Latinoamericana. Las posturas biologicistas se observan en el discurso de académicos colombianos y extranjeros, incluyendo a profesores universitarios estadounidenses. Por ejemplo, I. Perfecto y J. Vandermeer (24), afirman que el informe de la EPA no proporciona una adecuada evaluación de los riesgos ambientales planteados por el uso del glifosato en el programa de erradicación en Colombia. En su opinión, hay suficiente evidencia científica sobre los productos químicos usados en la fumigación aérea de coca, y que la forma de aplicación en Colombia plantea riesgos sobre el ambiente, que deberían ser evaluados antes de causar daños irreversibles.

Un ejemplo de las aproximaciones contextuales puede verse en un texto de dos profesoras de la Universidad Nacional de Colombia; ellas señalan que entre los problemas de mayor impacto negativo para la salud de los colectivos se destacan: i) la falta de provisión y alteraciones en el consumo y tipo de alimentos; ii) las condiciones de hacinamiento de toda aquella población que continúa en las zonas de fumigación y a la cual le han arrasado sus bienes; iii) las condiciones de hacinamiento y sanitarias de los desplazados por las fumigaciones, y iv) las condiciones psicosociales: Angustia, estrés, miedo, desolación, desintegración y desarraigo social. En su escrito intentan explicar algunos procesos y condiciones que podrían afectar la salud de los residentes en las zonas fumigadas (25). Pese a sus interesantes planteamien- 
tos teóricos, en ningún momento se señala un efecto directamente asociado con los plaguicidas, y más bien usan el determinismo social propio de su aproximación (26).

La aproximación ambientalista

Muchos grupos ambientalistas se han manifestado en contra de la fumigación de los cultivos ilícitos con plaguicidas. Como ejemplo se puede citar el discurso de Elsa Nivia de Rapalmira, en la Conferencia "Las guerras en Colombia: Drogas, armas y petróleo” en Davis, California. En ésta se presentan algunos estudios toxicológicos del Roundup ${ }^{\circledR}$ en animales y humanos, y se señala que puede existir una altísima absorción de Roundup Ultra ${ }^{\circledR}$ debido a las propiedades de los surfactantes (3). Esta es una revisión muy coherente, que utiliza diversas fuentes científicas para justificar su postura de no uso de plaguicidas para el control de cultivos ilícitos. Lo más interesante de este informe es la forma como la autora, utilizando muchas fuentes, logra evidenciar que la mezcla utilizada en Colombia aún no ha sido estudiada adecuadamente, y señalar las limitaciones e incongruencias de los estudios científicos sobre el tema. Los argumentos esbozados en los discursos ambientalistas no son muy diferentes a los que se exponen en otros escenarios, como en la agricultura, donde también se usan plaguicidas.

Discursos de los defensores de derechos humanos

Varios organismos no gubernamentales de derechos humanos, así como la Defensoría del Pueblo, se han manifestado en contra de las fumigaciones. Por ejemplo, en una carta enviada por varias organizaciones al Secretario de Estado estadounidense Colin Powell (27), se expone la preocupación por la situación, y se argumenta que las fumigaciones violan las leyes colombianas y están en contra del espíritu de las condiciones legales aprobadas por el senado estadounidense. En su carta no se llega a establecer nexos causales claros con los plaguicidas.

Los nexos entre salud y derechos humanos es un tema de muy reciente interés en salud pública. Una de las principales premisas es que las violaciones a los derechos humanos afectan la salud humana, principalmente cuando son severas, generalizadas o prolongadas (28), como es el caso de las fumigaciones de cultivos ilícitos. Sin embargo, no hay en la actualidad un marco general que permita comprender las relaciones entre el ambiente y la salud desde el punto de vista de los derechos humanos, debido a que no hay consenso sobre el significado de ambiente limpio, decente, saludable o seguro (28). Recientemente, algunos autores como Farmer \& Gastineau (29) indican 
que para entender estas relaciones es necesario verlas desde la perspectiva de las disciplinas contextuales, y no simplemente desde el punto de vista legal aferrado al Artículo 25 de la Declaración de los Derechos Humanos. De esta manera las desigualdades sociales, basadas en cualquier forma de discriminación, son "patologías del poder" que participan en la determinación de las condiciones de salud.

Una postura unificada en contra de las fumigaciones

En una carta enviada a Mary Robinson, Alta Comisionada de las Naciones Unidas para los Derechos Humanos (31), por varias personas y grupos sociales se puede tener una visión general integradora de quienes rechazan las fumigaciones de cultivos ilícitos en Colombia. Allí, el problema de los efectos adversos sobre la salud humana no es el fundamento de la crítica; más bien éste radica en el atentado a los derechos humanos de los residentes en las áreas fumigadas. De esta manera la violación de estos derechos es la forma de enlace entre el programa de erradicación de cultivos ilícitos y los efectos adversos sobre la salud humana.

Es interesante notar que esta carta finaliza con un llamado al principio de precaución (32), lo que significa que se sugiere tomar medidas preventivas aun sin tener clara la relación causa-efecto entre plaguicidas y salud. Esto requeriría, durante su implantación, utilizar la metodología científica y promover el uso de la tecnología en un contexto de transparencia y participación democrática. Como se puede inferir, esta podría ser una de las opciones posibles para intentar solucionar esta controversia, si se hace de una manera donde los diversos actores sociales estén realmente involucrados.

\section{DISCUSIÓN}

Del análisis realizado queda claro que no hay antecedentes en la forma de uso de los plaguicidas en los programas de erradicación de cultivos ilícitos en Colombia. Las evidencias aportadas por los gobiernos colombiano y estadounidense no son suficientes para descartar relaciones causales y tomar decisiones, máxime cuando los métodos científicos convencionales tienen limitaciones, por lo que se requiere complementarlos con otras aproximaciones (32). De manera similar, los otros actores tampoco muestran contundentemente que los plaguicidas tengan efectos sobre la salud humana, y más bien se vislumbra que, para formular este nexo causal, requieren de explicaciones complejas e indirectas, íntimamente relacionadas con el contexto sociopolítico en el que ocurre el debate. 
En un debate como éste, ¿qué papel puede tener la ciencia para intentar dirimirlo? Existe literatura científica que puede servir como fundamento para tomar decisiones. Sin embargo, debido a que los conocimientos de salud ambiental se originan principalmente en los países desarrollados, su aporte para solucionar la controversia puede verse como "importación de conocimiento" (33). Por esto, algunos actores sociales podrían decidir aceptar o no los argumentos científicos. Para complicar más el debate, algunos analistas han comparado la situación descrita con lo ocurrido en la guerra de Vietnam, cuando el ejército estadounidense usó el Agente Naranja (9) como parte de su estrategia militar. Es decir, que las fumigaciones son una forma de combatir a los grupos insurgentes, al provocar el desplazamiento de las poblaciones que podrían de alguna manera colaborar con la guerrilla (34). Allí también se presentaron controversias respecto a los efectos sobre la salud (35), y se pudo evidenciar que el "modelo médico" es incapaz de comprenderlo.

En conclusión, no se vislumbra una solución a corto o mediano plazo de este problema. Los gobiernos colombiano y estadounidense pretenden eliminar el contexto social del análisis para centrarse en la relación glifosatoefecto sobre la salud, mientras los críticos del programa de erradicación tienen una postura ecológica y/o social en la que todos los factores se encuentran asociados mediante complejas relaciones no fácilmente identificables. Este último enfoque enfatiza la determinación social de la enfermedad o interpreta la relación plaguicida-efecto adverso, mediada por la violación a los derechos humanos. Una posible solución es optar por herbicidas menos tóxicos o usar agentes biológicos. Sin embargo, la experiencia obtenida cuando se intentó usar el Fusarium oxysporum, indica que esta propuesta no será aprobada por varios actores sociales.

Ante esto, una solución muy acorde con los conceptos de la salud pública, podría ser el aceptar el principio de precaución (32). De esta manera se podrían eliminar completamente los efectos sobre la salud que han sido asociados con el glifosato. Sin duda alguna cualquier decisión que sobre esto se tome dependerá más de consideraciones políticas, que del conocimiento y de las evidencias que pueda enseñar la ciencia

\section{REFERENCIAS}

1. Joyce S. Environmental casualties of the war on the drugs. Environ. Health Perspect 1999; 107:A74-A77. 
2. Defensoría del Pueblo de Colombia. La ejecución de la estrategia de erradicación aérea de cultivos ilícitos, con químicos, desde una perspectiva constitucional. Bogotá: Defensoría del Pueblo; 2002.

3. Nivia E. Las fumigaciones aéreas sobre cultivos ilícitos si son peligrosas. Algunas aproximaciones, 2001. [Internet] Disponible en: http://www.biodiversiddla. org/documentos/documentos147.htm. Consultado el 7 de Enero de 2003.

4. Garvin T. Analytical paradigms: The epistemological distances between scientists, policy makers, and the public. Risk Anal 2001; 21:443-455.

5. Brown P. Qualitative methods in environmental health research. Environ. Health Perspect 2003; 111:1789-1798.

6. White-Riley M, Stoll CS. Content analysis. In: Sills DL (Ed.). International Encyclopedia of the Social Sciences Vol. 3. United States of America: The Macmillan Company \& The Free Press; 1968:371-377.

7. Goetz JP, Lecompte MD. Etnografía y diseño cualitativo en investigación educativa. Madrid: Morata; 1988.

8. Suppes P. Qualitative theory of subjective probability. In: Wright G, Ayton P (editors). Subjective probability. Chichester: John Wiley; 1994.

9. Massey R. The "drug war" in Colombia: Echoes of Vietnam. J. Public Health Pol 2001; 22:280-285.

10. Tenembaum D. Coca-killing controversy. Environ. Health Perspect 2002; 110 (5): A236.

11. Delor F, Hubert M. Revisiting the concept of "vulnerability". Soc. Sci. Med 2002; 50:1557-1570.

12. Chambers R. Rural development: Putting the last first. London: Longman; 1983.

13. Acción Ecológica. Reporte de la investigación de los impactos de las fumigaciones en la frontera ecuatoriana, 2001. [Internet] Disponible en: http:// www.accionecologica.org/fumigaciones_pc2_1.htm. Consultado el 12 de Febrero de 2004.

14. Maldonado A, Gallardo A, Alvarez T, Pazmiño JL, Reyes D, Chiriboga G, et al. Impactos en Ecuador de las fumigaciones realizadas en el Putumayo dentro del Plan Colombia Octubre - 2002 (Informe Misión de Verificación).

15. Heyman B, Henriksen M, Maughan K. Probabilities and health risks: A qualitative approach. Soc. Sci. Med 1998; 47:1295-1306.

16. Maldonado G, Greenland S. Estimating causal effects. Int. J. Epidemiol 2002; 31:422-429.

17. Winship C, Morgan SL. The estimation of causal effects from observational data. Annu. Rev. Sociol 1999; 25:659-706.

18. Grimes DA, Schulz KF. Descriptive studies: What they can and cannot do. Lancet 2002; 359:145-149.

19. Clínica Uribe Cualla. Estudio de las denuncias de daños a la salud relacionadas con la erradicación aérea en Colombia (Informe Final). Bogotá; 2001.

20. United States Environmental Protection Agency. U.S. Environmental Protection Agency Office of Pesticide Programs details of the consultation for department of state use of pesticide for coca eradication program in Colombia, August 2002. [Internet] Disponible en: http://www.state.gov/g/ inl/rls/rpt/aeicc/13237.htm. Consultado el 15 de Enero de 2003. 
21. Spurgeon A. Models of unexplained symptoms associated with occupational and environmental exposures. Environ. Health Perspect 2002; 110(Suppl.4): 601-605.

22. Rosenstock L, Lee LJ. Attacks on science: The risks to evidence-based policy. Am. J. Public Health 2002; 92:14-18.

23. Fuerzas Armadas Revolucionarias de Colombia. Comunicado, 2001. [Internet] Disponible en: http://six.swix.ch/farcep/Comunicados/2001/mar0801a. htmil. Consultado el 12 de Febrero de 2004.

24. Perfecto I, Vandermeer J. Letter to the Honorable Member of Congress, 2003. Disponible en: http://amazonalliance.org/scientific/pv.pdf. Consultado el 20 de Febrero de 2004.

25. García MC, Mejía NE. El impacto de las fumigaciones aéreas, 2003. [Internet] Disponible en: http://www.mamacoca.org/ed-especial1/tcap05.htm. Consultado el 18 de Enero de 2004.

26. Laurell AC. Social analysis of collective health in Latin America. Soc. Sci. Med 1989; 28:1183-1191.

27. Witness for Peace. Letter to the honorable Colin Powell, 2003. [Internet] Disponible en: http://witnessforpeace.org/colombia/fumigacion_letter. htmol . Consultado el 20 de Febrero de 2004.

28. Mann JM, Gostin L, Gruskin S, Brennan T, Lazzarini Z, Fineberg HV. Health and human rights. Health Hum. Rights 1994; 1: 7-24.

29. Center for Economic and Social Rights. Rights violations in the Ecuadorian Amazon. Health Hum. Rights 1994; 1:83-100.

30. Farmer P, Gastineau N. Rethinking health and human rights: Time for a paradigm shift. J. Law Med. Ethics 2002; 30:655-666.

31. Letter to Mary Robinson, UN High Commissioner for Human Rights, 2003. [Internet] Disponible en: http://mamacoca.org/Carta_mary_robinson_ fumigacion_aerea_en.htm.12. Consultado el 16 de Enero de 2004.

32. Kriebel D, Tickner J, Epstein P, Lemons J, Levins R, Loechler EL, et al. The precautionary principle in environmental science. Environ. Health Perspect 2001; 109:871-876.

33. Smith KR. Environmental health - for the rich or for all? Bull. WHO 2000; 78:1156-1157.

34. González M. The poisoned embrace: Plan Colombia and the expansion of imperial power. Int. Socialism J 2001. [Internet] Disponible en: http://www. pubs.socialistreviewindex.org.uk/isj93/gonzalez.htm. Consultado el 10 de Febrero de 2003.

35. Hall W. The logic of a controversy: The case of Agent Orange in Australia. Soc. Sci. Med 1989; 29:537-544. 